\title{
GASTROENTEROLOGY
}

\section{Incidence, endoscopic morphology and distribution of metastatic lesions in the gastrointestinal tract}

\author{
Shu-Chen Wei, ${ }^{*}$ Wei-Chih Su, ${ }^{\dagger}$ Ming-Chu Chang, ${ }^{*}$ Yu-Ting Chang, ${ }^{*}$ Cheng-Yi Wang ${ }^{*}$ and Jau-Min Wong ${ }^{*}$ \\ *Department of Internal Medicine, National Taiwan University Hospital and College of Medicine, and ${ }^{\dagger}$ Section of Gastroenterology, Taipei Buddhist \\ Tzu Chi General Hospital, Taipei, Taiwan
}

\author{
Key words \\ gastrointestinal tract, incidence, location, \\ metastasis, morphology. \\ Accepted for publication 14 March 2006.

\section{Correspondence} \\ Dr Jau-Min Wong, Department of Internal \\ Medicine, National Taiwan University Hospital \\ and College of Medicine, No. 7 Chung-Shan \\ South Road, Taipei, Taiwan. \\ Email: jmwong@ha.mc.ntu.edu.tw
}

\begin{abstract}
Background and Aim: Metastasis rarely occurs in the gastrointestinal tract (GIT). However, as progress regarding the treatment of cancers has occurred over recent years, the survival time of patients affected by advanced-stage cancers has increased. It could be expected that progressively more cancer patients with gastrointestinal symptoms would be presenting to gastroenterologists for diagnosis and further management. The aim of this study was to reveal the incidence, typical location and morphology of secondary tumors within the GIT.

Methods: A retrospective study was conducted at the National Taiwan University Hospital from 1 January 1994 to 31 December 2003 inclusive in order to review the available data pertaining to diagnosed GIT metastases. Only those patients who had been checked by endoscopy and confirmed by histopathology were included. Details regarding patient clinical information, demographic data, treatment, histopathology, and eventual outcome were reviewed, recorded and analyzed.

Results: The incidence of GIT metastases was found to be one upper GIT metastasis per 3847 upper GIT endoscopies and one lower GIT metastasis per 1871 colonoscopies. The common locations for metastases to occur were duodenum and stomach. The general morphology of the observed metastatic lesions of the digestive tract identified them, mostly, as single and primary (mucosa-origin) carcinoma-like lesions. The survival interval from the diagnosis of GIT metastasis to patient death was statistically significantly longer in the aggressive-treatment group compared to the conservative-treatment group (KaplanMeier, $P=0.0004)$.

Conclusions: Although metastatic lesions in the GIT are rare, they do occur. They usually present as single and primary carcinoma-like lesions, with the stomach and duodenum most commonly involved.
\end{abstract}

\section{Introduction}

Endoscopy has been part of the clinician's effective diagnostic armamentarium for decades. Certainly the ever-increasing frequency of use of this diagnostic modality, and the relative experience of the operator, have contributed to the progressively increasing popularity, ease of use and application of endoscopy to diagnose primary malignant lesions in the gastrointestinal tract (GIT), by endoscopically revealed morphology and/or by biopsied-tissue acquisition for subsequent histopathological study. To the best of our knowledge, however, for the identification of secondary malignant (metastatic) lesions within the GIT, it would appear that, for endoscopists, literature-reported experiences are limited, and relevant studies are few, being typically only case reports or case studies.
At least three possibilities could be used to theoretically explain such a situation. First, secondary malignant lesions rarely occur within the GIT. In 1993, Madeya et al. reported, as a result of a questionnaire survey, that one duodenal metastasis was verified amongst 3477 upper gastrointestinal endoscopies and no metastatic tumor was verified amongst 1634 examinations of the colon and rectum. ${ }^{1}$ Even with the benefit of autopsy results, the incidence of metastatic lesions in the esophagus has been reported to range from 0.3 to $6.1 \%,{ }^{2-5}$ and in the stomach the incidence ranged from 0.2 to $5.4 \%{ }^{6,7}$ To date, to the best of our knowledge, no study of the autopsy results for metastatic lesions in the colon, and estimates of the incidence of such, have been identified. Second, most patients who have been reported to have metastatic lesions in the GIT have also had a number of other sites of metastases. ${ }^{5}$ Therefore, for most patients at the time of referral for diagnosis 
and/or lesion management of GIT metastasis, the affected patients' general condition was poor, which might contribute to the reduced likelihood of acceptance, by the patient and/or their family, of subsequent therapeutically intended attempts at endoscopic study and treatment. The third possible explanation for such a perceived paucity of literature-cited examples of GIT metastasis management is that the major tool for diagnosing GIT lesions would appear to have progressively changed over the last couple of decades. Those available larger case studies reported in the literature focusing on a discussion of metastatic tumors of the GIT that we were able to review were mostly examples of a diagnosis performed by autopsy, surgical intervention and/or radiology, ${ }^{3,5,6,8,9}$ there being only a few case reports available that were described from the view of endoscopists. ${ }^{10-15}$

As a consequence of the progress made regarding either surgical or chemotherapeutic management of cancer over recent years, both the prognosis and survival time for cancer patients would appear to have improved notably. ${ }^{16,17}$ Lesions that previously would only have been likely to have been revealed by autopsy, may present to us in our daily practice nowadays and may be able to be relatively easily identified by current technology. Such an outcome would suggest that it could be expected that progressively more and more cancer patients with gastrointestinal symptoms would be likely to present to gastroenterologists for diagnosis and further management, and that the incidence of the diagnosis, by endoscopy, of secondary tumors of the GIT will likely increase.

The aim of this study was to investigate patient details of the incidence, location and the morphology of secondary tumors within the GIT at a tertiary medical center, by reviewing relevant patient chart records deriving from the preceding 10 years. We hope that the results of our study will be able to offer gastroenterologists sufficient information to become familiar with relevant details pertaining to metastatic tumors within the GIT.

\section{Methods}

A retrospective study was approved by Research Ethics Committee of the National Taiwan University Hospital and was conducted to review the data deriving from patients who had been diagnosed with GIT metastases. In order to obtain a more complete review of such cases, we searched two separate databases for relevant cases over the same 10-year period (from 1 January 1994 to 31 December 2003 inclusive) at the National Taiwan University Hospital. The first database was of endoscopic case studies (including upper GIT panendoscopy and colonoscopy). All cases that had been diagnosed as being metastatic lesions or suspicious metastatic lesions during endoscopic examination at the time were included in our study sample. The second database that we searched was a pathology database, for which those cases that had been diagnosed, by histopathology, as being metastatic lesions in the esophagus, stomach, duodenum and colorectal area were included. We then pooled the data from these two selected databases.

Finally, only those cases that had been checked with endoscopy and that had had the diagnosis confirmed with pathology as being examples of metastatic lesions of the GIT were included in this study. Metastatic lesions were defined by pathology using the histology picture compared to the primary site as well as by immunohistochemical staining. Further, those patients suffering from leukemia, malignant lymphoma with GIT involvement, intraabdominal cancer featuring direct invasion to the gastrointestinal tract and metastatic lesions that were obviously part of a general peritoneal invasion were excluded.

Patient clinical information, demographic data, treatment regimens, pathological details, and outcome details were reviewed, recorded and analyzed. For clearer classification of the metastatic lesions endoscopically, they were divided into either single- or multiple-lesion groups; and morphologically categorized as primary (mucosa-origin) carcinoma-like or submucosal tumor-like lesions. The criteria for submucosal tumor-like lesions are defined as sessile tumors with bridging folds, and the mucosa is mostly normal. The criteria for the primary (mucosa-origin) carcinomalike lesions are defined according to the Japanese classification of gastric carcinoma by the Japanese Gastric Cancer Association.

A survival curve was calculated using the Kaplan-Meier method. Differences in survival time for patients receiving aggressive treatment (e.g. surgical intervention, endoscopic hemostasis, intervention angiography and chemotherapy) versus those receiving conservative treatment (e.g. observation and palliative therapy) were analyzed using the Cox proportional hazard model to avoid confounding factors such as age, sex, primary sites of tumor and the coexistence of other metastasis. As a retrospective study, we could only divide the patients into aggressive and conservative treatment groups according to the patient records instead of making a definite indication. However, aggressive treatment would have been given to all patients with acceptable general condition unless the patient/family refused.

\section{Results}

A total of 42 cases of GIT metastasis ( 26 men, 16 women) were included in our study according to the specified inclusion criteria. Over this 10-year period, a total of 126966 upper GIT panendoscopies and 16838 colonoscopies were performed at our institution. One upper GIT metastasis was verified per 3847 upper gastrointestinal endoscopies, and one lower GIT metastasis was verified per 1871 colonoscopies. The age at time of primary cancer diagnosis ranged from 19.5 to 83 years, with the mean being 55.0 years. The age of diagnosis of GIT metastasis ranged from 20.9 to 83 years, with the mean age being 57.3 years. The survival period from diagnosis of GIT metastasis ranged from less than 1 month to 42 months, with a mean of 5.64 months.

Three patients in the series received repeated panendoscopies but no patients received repeated colonoscopies. The first patient had persistent bleeding, and underwent repeated scope for hemostasis but this still failed. Angiography with embolization had been arranged but the patient died of massive bleeding. The second patient had much coffee ground substance during the first panendoscopy, and the tissue proof by biopsy was obtained at the second panendoscopy. The third patient had diagnosis of the metastatic lesion in GIT during the first panendoscopy, and the follow-up scope 6 months after chemotherapy showed a good response to treatment.

Amongst the 42 patients with GIT metastasis, 31 (73.8\%) had metastasis elsewhere. The interval from the diagnosis of the initial cancer to the diagnosis of GIT metastasis ranged from 0 days (both diagnoses at the same time) to 9.5 years, with a 
mean \pm standard deviation of $2.3 \pm 2.6$ years. The categorized indications for endoscopic investigation are listed in Table 1, with GIT bleeding being the most common and abdominal pain as the second most common indication.

The primary sites of cancer are shown in Table 2, with breast cancer, lung cancer and hepatocellular carcinoma being the three leading cancers, in that order, to metastasize to the GIT. We divided the primary cancers on the basis of metastasis to either upper or lower GIT, noting that lung cancer was the most common primary cancer to metastasize to the upper GIT (Fig. 1a). Breast cancer and transitional cell carcinoma demonstrated equal highest propensity to metastasize to the lower GIT (Fig. 1b).

In the context of the distribution of the metastatic locations within the GIT, the duodenum $(44.2 \%)$ was the most common and the stomach $(27.9 \%)$ the second most common sites for GIT metastatic lesions to be located (Table 3) (Fig. 1). The majority of the metastatic lesions located within the GIT were solitary (26 patients, $61.9 \%$ ) and primary (mucosa-origin) carcinoma-like lesions (38 patients, 90.5\%). Amongst our 42 patients, 25 (59.5\%) were successfully diagnosed, endoscopically, as having metastatic lesions, 12 patients $(28.6 \%)$ as having primary cancer, and five patients $(11.9 \%)$ as having benign lesions.

Subsequent to the diagnosis of metastatic GIT lesions having been made, four patients underwent surgery, one patient under-

Table 1 Indications for endoscopy in 42 patients with gastrointestinal tract metastasis

\begin{tabular}{lcc}
\hline Indication & $n$ & $\%$ \\
\hline Gastrointestinal tract bleeding & 25 & 59.5 \\
Abdominal pain & 8 & 19.0 \\
Diarrhea & 3 & 7.1 \\
Metastasis for search primary site & 3 & 7.1 \\
Gastrointestinal tract obstruction & 1 & 2.4 \\
Increased CEA & 1 & 2.4 \\
Suspicious lesion by trans-rectal echo & 1 & 2.4 \\
Total & 42 & 100 \\
\hline
\end{tabular}

CEA, carcinoembryonic antigen.

Table 2 Primary cancers of 42 patients with gastrointestinal tract metastasis

\begin{tabular}{lcc}
\hline Primary cancer & $n$ & $\%$ \\
\hline Breast cancer & 7 & 16.7 \\
Lung cancer & 7 & 16.7 \\
Hepatocellular carcinoma & 5 & 11.9 \\
Colorectal cancer & 5 & 11.9 \\
Gastric cancer & 3 & 7.1 \\
Melanoma & 2 & 4.8 \\
Sarcoma & 3 & 7.1 \\
Transitional cell carcinoma (prostate \& urinary bladder) & 3 & 7.1 \\
Unknown primary & 2 & 4.8 \\
Renal cell carcinoma & 1 & 2.4 \\
Germ cell tumor & 1 & 2.4 \\
Gall bladder cancer & 1 & 2.4 \\
Choriocarcinoma & 1 & 2.4 \\
Adrenal cortical carcinoma & 1 & 2.4 \\
Total & 42 & 100 \\
\hline
\end{tabular}

went angiography with transarterial embolization, one patient received endoscopic hemostasis, 11 patients received chemotherapy, and the remaining 25 patients received only conservative treatment. The condition-associated symptoms were able to be

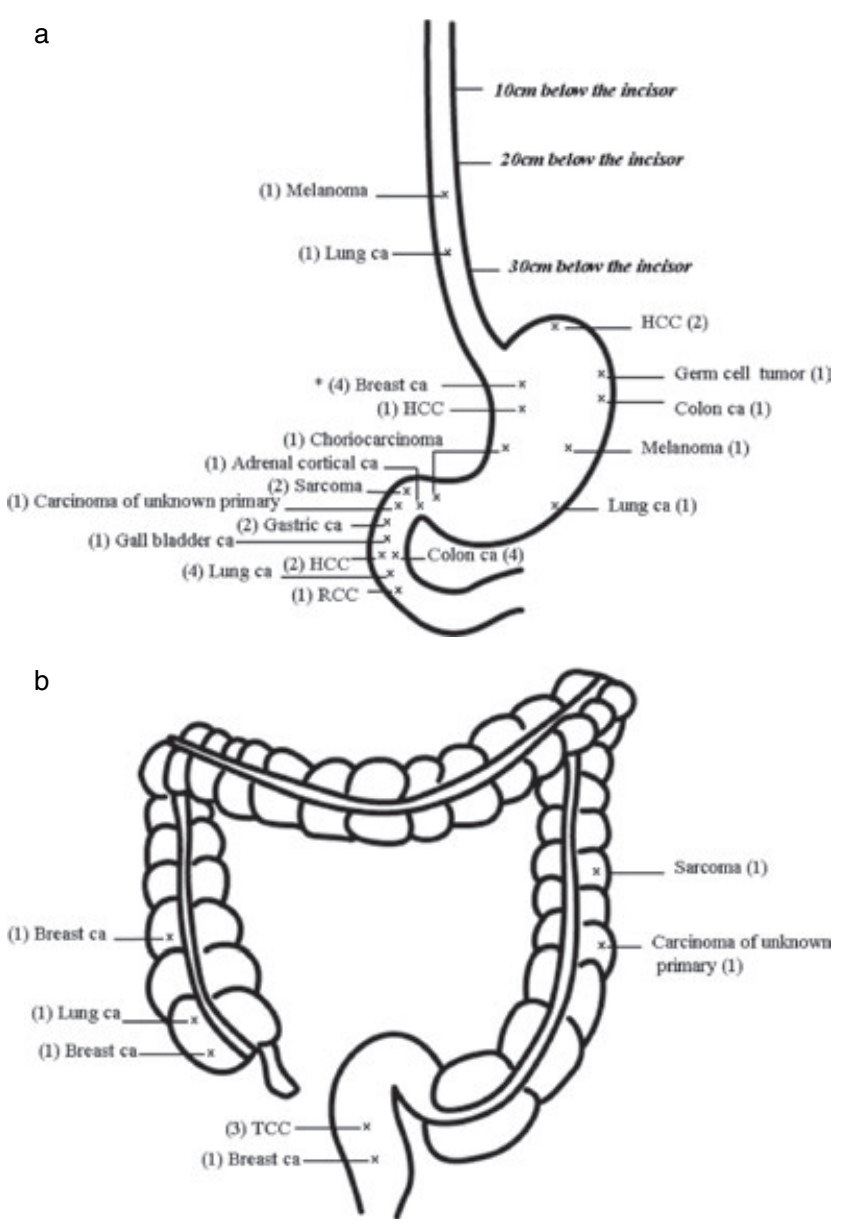

Figure 1 Distribution of gastrointestinal tract metastases with primary cancer labeled: (a) upper gastrointestinal tract; and (b) lower gastrointestinal tract. Number of patients is shown in parentheses. $\mathrm{Ca}$, cancer; HCC, hepatocellular carcinoma; RCC, renal cell carcinoma; TCC, transitional cell carcinoma. ${ }^{*}$ Two cases were of diffuse type lesions in the stomach.

Table 3 Locations of the gastrointestinal tract metastases

\begin{tabular}{lrr}
\hline Location & $n$ & $\%$ \\
\hline Upper gastrointestinal tract & 2 & 4.7 \\
$\quad$ Esophagus & 12 & 27.9 \\
Stomach & 19 & 44.2 \\
Duodenum & 1 & 2.3 \\
Efferent-loop & & \\
Lower gastrointestinal tract & 4 & 9.3 \\
$\quad$ Rectum & 5 & 11.6 \\
Colon & $43^{\dagger}$ & 100 \\
\hline Total &
\end{tabular}

${ }^{\dagger}$ One patient had both duodenum and stomach involvement. 


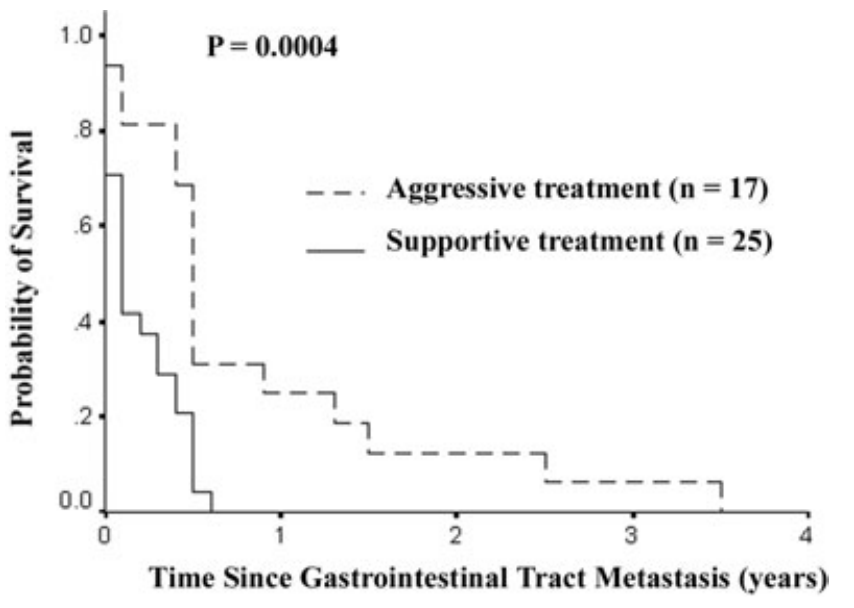

Figure 2 Survival curves of patients with gastrointestinal tract metastasis.

relieved for 21 patients $(50 \%)$, but this was not the case for the remaining patients.

Although no patients survived their conditions over the period that we reviewed their medical charts, the survival interval from the diagnosis of GIT metastasis to the date of patient death was significantly longer in the aggressive treatment group compared to the conservative treatment group (Kaplan-Meier, $P=0.0004$; Fig. 2). Cox proportional hazard modeling with survival as the dependent variable showed that only the treatment (aggressive $v \mathrm{~s}$ conservative, $P=0.044$ ) was significantly independently related, whereas age $(P=0.663)$, sex $(P=0.957)$, primary tumor site $(P=$ 0.943 ), metastatic tumor site (upper or lower GIT; $P=0.332$ ), and the coexistence of other metastasis $(P=0.944)$ were not.

\section{Discussion}

Previously considered a rare condition, ${ }^{1-8}$ to the best of our knowledge, there would not appear to be available any studies reported in the literature pertaining to the actual incidence of the diagnosis of GIT metastasis by endoscopic means. Only one result from a questionnaire survey of the staff of 34 medical clinics showed that one metastasis was verified amongst 3477 upper GIT endoscopic procedures, but not one lower GIT metastasis appeared to have been detected from amongst 1634 colonoscopies. ${ }^{1}$ To the best of our knowledge, our study is the first report of a retrospective study to disclose the incidence of GIT metastasis as verified by endoscopy, our results indicating that one upper GIT metastasis was verified per 3847 upper GIT endoscopies and one lower GIT metastasis was verified per 1871 colonoscopies. Our incidence of upper GIT metastasis as verified by endoscopy was similar to the result reported for a previous questionnaire survey by Madeya et al. ${ }^{1}$ Further, the low incidence of lower GIT metastasis in our study might also explain why the questionnaire survey by Madeya et al. did not reveal any examples of metastasis in this location.

Metastases may occur at virtually any site within the entire digestive tract. ${ }^{3-9,18,19}$ According to an autopsy report, most GIT metastases are observed in the stomach and in the small bowel, ${ }^{5}$ although, as our results have shown, the duodenum was the most common site at which metastases occurred, with the stomach being the second most common site. Clearly there exist differences between the results of the 1997 study of Mizobuchi et al. and our study. First, Mizobuchi et al.'s results originated from autopsy while our results derived from endoscopic examination. Second, the ethnicity of the study samples appeared to be different; that is, Japanese versus Taiwanese, respectively. Third, the primary tumor types may have been different to very different, which may have led to the involved metastatic sites also being different.

Lung cancer, breast cancer and melanoma were the three cancers reported to be most commonly associated with metastasis to the upper GIT. ${ }^{3,5,6,20}$ Our results showed that lung cancer, hepatocellular carcinoma, colorectal cancer and breast cancers were the cancers commonly associated with metastasis to the upper GIT. Melanoma as a primary lesion had been found to be associated with metastasizing to the upper GIT in our study but not so commonly as has been reported previously. ${ }^{3,5,6,20}$ Rather, from our study, hepatocellular carcinoma was found to be a primary lesion that was rather commonly associated with metastasizing to the upper GIT, whereas previously it appears that such an example had been reported as a single case study only. ${ }^{21}$ We speculate that such a difference might be explained by the rather high incidence of hepatocellular carcinoma in Taiwan as compared to Western countries, ${ }^{22}$ this lesion type being the most common cancer in Taiwan. ${ }^{23}$ Therefore, it would appear likely that for different countries, differences exist as regards cancer epidemiology, and that the primary cancer most commonly metastasizing to the digestive tract may differ from country to country or ethnicity to ethnicity.

For our endoscopically diagnosed cases, GIT metastases presented more frequently as solitary $(61.9 \%)$ rather than multiple $(38.1 \%)$ lesions. From our literature review, we did note one report of metastatic tumors to the stomach according to endoscopic and autopsy results; ${ }^{6}$ the results of that study listed solitary metastatic tumors for $65 \%$ of cases, and multiple lesions for $35 \%$ of cases, not dissimilar to our results. When considering the endoscopic appearance of the GIT metastasizing lesions, our results indicated that most tumors were primary (mucosa-origin) carcinoma-like lesions $(90.5 \%)$, a result which appeared to be quite different to that of a previous report, for which Oda et al. reported a submucosal tumor-like appearance of metastases $(51 \%)$ was more common than primary (mucosa-origin) carcinoma-like lesions (39\%). ${ }^{6}$ However, it should be remembered that Oda et al.'s results were based upon metastatic tumors to the stomach only, while our results were based upon metastatic tumors to the entire digestive tract.

According to our results, of the 42 patients, 25 (59.5\%) were successfully diagnosed, endoscopically, as having metastatic lesions. There were 12 patients $(28.6 \%)$ whose lesions had been diagnosed, endoscopically, as primary cancers, and the other five patients $(11.9 \%)$ were endoscopically diagnosed as having benign lesions. Such a result would suggest that if we had not taken a biopsy for these 17 patients, $40.5 \%$ of our total pool of patients would probably have had the diagnosis missed. Therefore, for patients with a history of any type of cancer, biopsy for histopathological study for any subsequently developing GIT lesion(s) detected endoscopically and communication with the pathologist as necessary are strongly recommended.

Although selection bias exists in such a retrospective study, we have analyzed our data using the Cox proportional hazard model 
to adjust for the confounding factors. The results showed that only the treatment (aggressive $v s$ conservative, $P=0.044$ ) was significantly independently related. Our results showed that although most patients with GIT metastases in our study had lesions that belonged to the advanced stage of disease status, and $73.8 \%$ of the patients reviewed in our study had the presence of metastases elsewhere, aggressive treatment for these GIT-metastasizing patients does still appear to be warranted. Clearly, such aggressive treatment did, mostly, relieve the symptoms of such metastasis, and the duration of patient survival was able to be prolonged significantly by such treatment by comparison to those patients who received conservative treatment only.

In conclusion, the incidence of GIT metastases was verified as being about one upper GIT metastasis per 3847 upper GIT endoscopies and one lower GIT metastasis per 1871 colonoscopies, with common locations for metastasis being duodenum and stomach. The morphologies of the metastatic lesions to the digestive tract were shown to be, mostly, single, primary (mucosa-origin) carcinoma-like lesions. According to our results, we suggest aggressive biopsy for cancer patients who have GIT lesions revealed endoscopically, and also aggressive treatment for relieving such patients' symptoms and attempting to prolong their survival.

\section{References}

1 Madeya S, Borsch G, Greiner L, Hahn HJ. [How frequent is the diagnosis of GI metastasis in an endoscopic patient sample in general internal medicine clinics? Results of a questionnaire survey of 34 medical clinics.] Leber Magen. Darm 1993; 23: 77-80, 83 (in German).

2 Toreson W. Secondary carcinoma of the esophagus as a cause of dysphagia. Arch. Pathol. 1944; 38: 82-4.

3 Mizobuchi S, Tachimori Y, Kato H, Watanabe H, Nakanishi Y, Ochiai A. Metastatic esophageal tumors from distant primary lesions: report of three esophagectomies and study of 1835 autopsy cases. Jpn J. Clin. Oncol. 1997; 27: 410-14.

4 Abrahms H, Spiro R, Goldstein N. Metastases in carcinoma: analysis of 1000 autopsied cases. Cancer 1950; 3: 74-85.

5 Telerman A, Gerard B, Van den Heule B, Bleiberg H. Gastrointestinal metastases from extra-abdominal tumors. Endoscopy 1985; 17: 99101.

6 Oda Kondo H, Yamao T, Saito D et al. Metastatic tumors to the stomach: analysis of 54 patients diagnosed at endoscopy and 347 autopsy cases. Endoscopy 2001; 33: 507-10.

7 Menuck LS, Amberg JR. Metastatic disease involving the stomach. Am. J. Dig. Dis. 1975; 20: 903-13.

8 Berger A, Cellier C, Daniel C et al. Small bowel metastases from primary carcinoma of the lung: clinical findings and outcome. Am. J. Gastroenterol. 1999; 94: 1884-7.

9 Agha FP. Secondary neoplasms of the esophagus. Gastrointest. Radiol. 1987; 12: 187-93.

10 Gurian L, Ireland K, Petty W, Katon R. Carcinoma of the cervix involving the duodenum: case report and review of the literature. $J$. Clin. Gastroenterol. 1981; 3: 291-4.

11 Holderman WH, Jacques JM, Blackstone MO, Brasitus TA. Prostate cancer metastatic to the stomach. Clinical aspects and endoscopic diagnosis. J. Clin. Gastroenterol. 1992; 14: 251-4.

12 Kimura H, Kinoshita S, Takahashi I. Gastric metastases from accessory breast cancer. Gastrointest. Endosc. 2002; 56: 566-7.

13 Kishino T, Yamaguchi Y, Yamagishi T et al. Submucosal tumor (SMT)-like esophageal squamous cell carcinoma with gastric metastasis. Hepatogastroenterology 2000; 47: 1581-4.

14 Taylor RR, Phillips WS, O'Connor DM, Harrison CR. Unusual intramural gastric metastasis of recurrent epithelial ovarian carcinoma. Gynecol. Oncol. 1994; 55: 152-5.

15 Friedman E, Kwan MR, Cummins L. Squamous cell carcinoma of the transverse duodenum. Gastrointest. Endosc. 1986; 32: 99-101.

16 Mayer RJ. Two steps forward in the treatment of colorectal cancer. $N$. Engl. J. Med. 2004; 350: 2406-8.

17 Hurwitz H, Fehrenbacher L, Novotny W et al. Bevacizumab plus irinotecan, fluorouracil, and leucovorin for metastatic colorectal cancer. N. Engl. J. Med. 2004; 350: 2335-42.

18 Ozuner G. Colonoscopic detection of a malignant melanoma metastatic to a tubular adenoma of the colon: report of a case. Dis. Colon Rectum 2002; 45: 1681-4.

19 Bender GN, Maglinte DD, McLarney JH, Rex D, Kelvin FM. Malignant melanoma: patterns of metastasis to the small bowel, reliability of imaging studies, and clinical relevance. Am. J. Gastroenterol. 2001; 96: 2392-400.

20 Moreto M. Diagnosis of esophagogastric tumors. Endoscopy 2003; 35: 36-42.

21 Kume K, Murata I, Yoshikawa I, Kanagawa K, Otsuki M. Polypoid metastatic hepatocellular carcinoma of the esophagus occurring after endoscopic variceal band ligation. Endoscopy 2000; 32: 419-21.

22 El-Serag HB. Hepatocellular carcinoma: an epidemiologic view. $J$. Clin. Gastroenterol. 2002; 35: S72-8.

23 Department of Health. Health and Vital Statistics, Taiwan. Taipei: San Min Book Co, 2003. 